\title{
Parameterization of a Coarse-Grained Model of Cholesterol with Point-Dipole Electrostatics
}

\author{
P. Siani ${ }^{a, *}$, H. Khandelia ${ }^{b}$, M. Orsi ${ }^{c}$, L.G. Dias $^{a}$
}

aDepartment of Chemistry, FFCLRP, University of São Paulo, Ribeirão Preto, SP, Brazil.

${ }^{b}$ MEMPHYS-Center for Biomembrane Physics, Department of Physics and Chemistry, University of Southern Denmark, Denmark.

${ }^{\mathrm{c}}$ Department of Applied Sciences, Faculty of Health and Applied Sciences, University of the West England (UWE Bristol), Bristol, United Kingdom. 


\begin{abstract}
We present a new coarse-grained (CG) model of cholesterol (CHOL) for the electrostatic-based ELBA force field. A distinguishing feature of our CHOL model is that the electrostatics is modeled by an explicit point dipole which interacts through an ideal vacuum permittivity. The CHOL model parameters were optimized in a systematic fashion, reproducing the electrostatic and nonpolar partitioning free energies of CHOL in lipid/water mixtures predicted by full-detailed atomistic molecular dynamics (MD) simulations. The CHOL model has been validated by comparison to structural, dynamic and thermodynamic properties with experimental and atomistic simulation reference data. The simulation of binary DPPC/cholesterol mixtures covering the relevant biological content of CHOL in mammalian membranes is shown to correctly predict the main lipid behavior as observed experimentally.
\end{abstract}




\section{INTRODUCTION}

Cholesterol, an organic amphiphilic molecule belonging to the group of natural steroid alcohols, plays an essential role in regulating the biophysical properties of cell membranes, such as ordering, lateral diffusion, and mechanical stability. Cholesterol has also been associated with lipid raft formation [1-12], membrane permeability [13-15], and lipid-protein and protein-protein interactions $[13,16-20]$ in biological membranes.

Computer models of cholesterol have been previously developed with full atomistic resolution. These models provide detailed microscopic insights into complex cholesterolphospholipid mixtures. Recently, simulation techniques have been employed to support experimental and theoretical findings regarding complex cholesterol-lipid mixtures [21-35]. Unfortunately, all-atom (AA) simulations are still far from the temporal and spatial scales of experimental studies. Consequently, supra-atomic coarse-grained (CG) simulations arise as an attractive alternative to explore such systems beyond scales achieved by atomistic simulations. Complex chemical structures like sterols, proteins, and lipids remain a major challenge in molecular modeling. Concerning parameterization of new CG models for biomolecules, two main methodologies exist. The first is the top-down approach, in which bulk thermodynamic and/or structural information is used to derive chemical building blocks for CG force fields [36-38]. Some degree of transferability is allowed, so a broad range of applications are possible [39-53]. The second is the bottom-up approach, in which effective CG potentials are derived from atomistic models. In particular, effective potentials for CG site-site interactions may be derived by using Force Matching (FM) [54,55], Iterative Boltzmann Inversion (IBI) [56,57], or the Inverted Monte Carlo (IMC) technique [58]. 
In the 1980s and 1990s, Ipsen and coworkers [59] and Miao and coworkers [31] developed some of the earliest CG models in an attempt to understand the behavior of cholesterolphospholipid mixtures. Because these models corresponded to two-dimensional model descriptors of phosphatidylcholine-cholesterol systems interacting by a phenomenological Hamiltonian, they allowed phosphatidylcholine-cholesterol phase diagrams to be constructed and coexistence lines between the So (solid, ordered-chain), Lo (liquid, ordered-chain), and Ld (liquid, disorderedchain) phases to be determined.

Murtola and coworkers [60] took a step forward when they proposed a two-dimensional model to represent the dipalmitoylphosphatidylcholine-cholesterol (DPPC/cholesterol) mixture. These authors obtained the effective interaction potential by the IMC technique from AA-MD simulations, which allowed complex lipid mixtures to be exploited on a large length scale. The original model was further improved in subsequent work [61,62].

In the same spirit, Khelashvili and co-workers [63] used a self-consistent mean-field (SCMF) model based on MD simulations to study a two-dimensional representation of the DPPC/cholesterol model system. Their study enabled complex lipid models to be simulated on large spatial (500 nm in lateral size) and temporal (around dozens of microseconds) scales. De Meyer and Smit also studied both cholesterol and phospholipids by means of dissipative particle dynamics (DPD) via CG models. This particular work provided a new understanding of DMPC/cholesterol mixture phase diagrams [64].

Hadley and McCabe proposed a new cholesterol model for CG simulations based on the IBI technique [65]. This model correctly described the cholesterol crystalline solid state approximately 180 times faster than analogous AA simulations. The authors also explored the self- 
assembly of a set of skin lipids and investigated how the cholesterol content affected these systems [66].

Izvekov and Voth [54] introduced a new view of CG based on the FM technique. They mapped dimyristoylphosphatidylcholine (DMPC), cholesterol, and water molecules into fewer site bead representations - thirteen beads, four (or seven) beads, and one bead, respectively. CG-MD simulations of a hydrated DMPC bilayer containing cholesterol molecules were 50-100 times faster than AA-MD simulations of a system of equivalent size.

Marrink and co-workers [36] proposed a new version of the popular MARTINI Force Field (so-called MARTINI-FF 2.0). They suggested a protocol to model ring structures with specific applications to cholesterol. Therefore, the cholesterol molecule was modeled with a total of eight beads - six beads for the rigid sterol body and two beads for the hydrocarbon tail. These authors also simulated mixtures containing DPPC and cholesterol; they covered a broad range of cholesterol content, from $0 \mathrm{~mol} \%$ to $60 \mathrm{~mol} \%$. MARTINI-FF development stimulated subsequent research into biomembrane mimetic systems containing cholesterol [36,67-83]. Moreover, Daily and co-workers carried out a systematic optimization of structural parameters based on atomistic reference simulations, to improve MARTINI-FF for lipids [84]. This revised model predicted cholesterol ordering and thickening effects on the palmitoyloleoylphosphatidylcholine (POPC) bilayer better as compared to the original MARTINI-FF.

Shen and co-workers [85] recently proposed a new CG model for cholesterol based on the CAVS-FF, in which two opposite charges embedded into different sites describe the cholesterol electrostatics. This model correctly predicts the effects of cholesterol concentration on membrane dipole potential. 
Here, we present a point-dipole CG model for cholesterol (designated CHOL hereafter) that is consistent with ELBA-FF (ELectrostatic BAsed Force Field) [37]. We also provide an ELBA-FF extension for the DPPC lipid model, derived from such a set of optimized parameters available for the POPC lipid model [86]. A key feature of ELBA-FF is that it describes electrostatic interactions through charges and dipoles rather than using the standard practice of most CG FFs which employ point charge interactions shielded by an effective dielectric constant or incorporates it all into a short range potential. Thus, ELBA-FF is especially suitable to model molecules with different polar groups because it provides a realistic mapping of their polar moieties through explicit point dipoles. In particular, ELBA-FF allows functional mapping of CHOL polar moieties through point dipoles.

This paper is organized as follows. The first section (Computational Methodology) reports the MD simulation details and analysis protocols. The subsequent sections address CHOL model parameterization based on physicochemical properties obtained from experimental and atomistic reference data. We then compare our predictions for the CHOL and DPPC self-diffusion coefficients and the free energy of CHOL desorption membrane partitioning in pure and CHOLrich DPPC bilayers to the AA and CG reference simulations. 


\section{COMPUTATIONAL METHODOLOGY}

\subsection{Coarse-grained simulations}

MD simulations were carried out with LAMMPS (http://lammps.sandia.gov) version February 16, 2016 [87]. Initial coordinates for the binary DPPC bilayer systems at 10, 20, 30, 40, and $50 \mathrm{~mol} \%$ CHOL content (amounting to a total of 128 lipids) were generated with the MOLTEMPLATE program [88]. Two water layers were placed at the top and bottom of the lipid bilayer systems, which corresponded to a hydration level equal to 50 water molecules per lipid molecule, in agreement with typical experimental conditions. Additionally, a simulation box containing one CHOL molecule solvated by 8000 ELBA water molecules and another simulation box containing 1000 CHOL molecules were also constructed.

VMD [89] and MEMPLUGIN [90] were used to visualize and to analyze the MD trajectories. Shifted-force interactions for the Lennard-Jones and electrostatic potentials were used with truncation at a cutoff radius of $12 \AA$. Integration time steps of $10 \mathrm{fs}$ and 8 fs were employed in the absence and presence of CHOL molecules, respectively. The velocity-Verlet integrator [91] was used for all the CG MD simulations. The simulation temperature was maintained at $303 \mathrm{~K}$ by using a Langevin thermostat with a damping coefficient of $1 \mathrm{ps}^{-1}$. Semi-isotropic pressure coupling was used to control the pressure at 1 atm [92] with an oscillation period of 1 ps and a compressibility of $4.6 \times 10^{-5} \mathrm{~atm}^{-1}$. After initial energy minimization, followed by an equilibration step for 0.5 microseconds, production runs reached 1.6 microseconds for all of the studied systems. Original ELBA-FF parameters for water and lipids were taken from Orsi and Essex [37]. The new set of ELBA-FF parameters for CHOL is available in the Supplementary Information (SI).

\subsection{Atomistic simulations}


AA MD simulations were carried out with the NAMD 2.9 program [93] using CHARMM36-FF for lipids [94,95] and the TIP3P water model [96]. The Particle Mesh Ewald (PME) method [97] was employed for long-range electrostatic interactions, and 1-4 non-bonded interactions were turned on. The initial configuration for the hydrated DPPC bilayer system containing a CHARMM36-based CHOL molecule $[94,95]$ was constructed with CHARMM-GUI Membrane Builder [98]. In addition, a simulation box containing 32154 TIP3P water molecules solvating a CHARMM36 CHOL molecule $[94,95]$ was generated by using Solvate tools of the VMD program [89]. Electrostatic and Lennard-Jones potentials were used with a cut-off radius of $12 \AA$. The SETTLE algorithm [99] was used for rigid water molecules, whereas Newton's equations of motion were solved by means of the MTS integrator Verlet-I [100] with a 2 fs time step.

\subsection{Alchemical free energy - Thermodynamic Integration (TI)}

The electrostatic term of the solvation free energy was calculated with the aid of Thermodynamic Integration (TI), as implemented in both LAMMPS [87] and NAMD [93] packages. In particular, the electrostatic free energy was estimated through the following equation:

$$
\Delta G=\int_{0}^{1}\left\langle d U_{e l} / d \lambda\right\rangle_{\lambda} d \lambda
$$

where $U_{e l}$ is the electrostatic potential energy, and $\lambda$ is the coupling parameter with limits of integration between 0 and 1 . The $\langle d U e l / d \lambda\rangle_{\lambda}$ term is the average derivative of the electrostatic potential energy as a function of $\lambda$.

Numerical integration through a cubic spline interpolation over ten equally spaced $\lambda$ points was used to compute the electrostatic free energy. The CHOL molecule was harmonically 
restrained by a force constant equal to $100 \mathrm{kcal} / \mathrm{mol} \AA^{2}$ in the mass center of both bulk water and the DPPC bilayer. This allowed us to evaluate the electrostatic free energy difference of CHOL desolvation in the membrane.

The statistical uncertainty of the means was estimated by block averaging over the entire $\lambda$ interval. Standard errors were plotted as a function of the block length. The plateau value calculated by averaging values in the limit of infinite block length gave an estimate of the 'true' standard error [101,102].

\subsection{Mean force potential - Adaptive Biased Force (ABF)}

Free energy profiles were calculated by the Adaptive Biasing Force (ABF) approach [103-105] implemented in both the LAMMPS [87] and NAMD [93] programs. By definition, the average force exerted on the transition coordinate, $\xi$, can be related to the free energy first derivative according to:

$$
\frac{d A(\xi)}{d \xi}=\left\langle\frac{\delta U(x)}{\delta \xi}-\frac{1}{\beta} \frac{\delta \ln |J|}{\delta \xi}\right\rangle_{\xi}=-\left\langle F_{\xi}\right\rangle_{\xi}
$$

where $|\mathrm{J}|$ is the Jacobian determinant to transform the Cartesian coordinates to generalized coordinates, and $\left\langle F_{\xi}\right\rangle_{\xi}$ denotes the cumulative average of the instantaneous force $F_{\xi}$ in bins with the size of $\delta \xi$. In the $\mathrm{ABF}$ approach, the force applied to overcome energetic barriers along the reaction coordinate can be defined as:

$$
F^{A B F}=-\left\langle F_{\xi}\right\rangle_{\xi} \nabla_{x} \xi
$$


where $\left\langle F_{\xi}\right\rangle_{\xi}$ denotes the cumulative mean of $F_{\xi}$, calculated for each step of MD simulations. $F^{A B F}$ represents the biasing force that ideally reaches zero in its convergence.

For $\mathrm{ABF}$ calculations, the reaction coordinate was discretized in consecutive windows with size equal to $5 \AA$. A "non-charged" version (only Lennard-Jones interactions are active) of ELBAbased and CHARMM36-based CHOL molecules $[94,95]$ was dragged from bulk water toward the bilayer center in their respective systems. A constant force equal to $10 \mathrm{kcal} / \mathrm{mol} \AA^{2}$ in increments $\delta \xi$ of $0.1 \AA$ (ABF-AA simulations) and $1.0 \AA$ (ABF-CG simulations) was employed.

The statistical error of the mean force for each particular bin can be obtained from the standard error of the mean according to the following relation [106]:

$$
\operatorname{Err}\left[\left\langle F_{\xi}\right\rangle_{i}\right]=\sqrt{\frac{\left\langle\Delta F_{\xi}^{2}\right\rangle_{i}}{m_{i}}}
$$

where $\left\langle\Delta F_{\xi}^{2}\right\rangle_{i}$ represents the variance of the instantaneous force collected to bin $i$, and $m_{i}$ is the independent samples. The number of independent samples $m_{i}$ can be obtained by the product between the total number of simulation steps, $n_{i}$, and the MD time step, $\Delta t$, divided by the autocorrelation time, $\tau_{i}$, of the instantaneous force, $m_{i}=n_{i} \Delta t / \tau_{i}$. In particular, $\tau_{i}$ values for each specific window were obtained by non-linear fitting of an exponential function $e^{-t / \tau}$. The Bienaymé formula [107] was used to calculate the error of the sum of mean forces for points in different windows: 


$$
\operatorname{Err}\left[\Delta A_{a \rightarrow b}\right]=\delta \xi\left(\sum_{i=i_{a}}^{i_{b}} \frac{\tau_{i}}{n_{i} \Delta t}\left\langle\Delta F_{\xi}^{2}\right\rangle_{i}\right)^{1 / 2}
$$

\subsection{Thermodynamic calculations}

We obtained the total free energy difference of CHOL partitioning in both pure and CHOL-rich DPPC bilayers by employing the thermodynamic cycle shown in Scheme 1.

Scheme 1. Thermodynamic cycle employed to calculate the total free energy of CHOL partitioning in lipid bilayers:

$$
\begin{aligned}
& \mathrm{CHOL}_{(\mathrm{n})}^{\text {(charged) }} \stackrel{\Delta \mathrm{G}_{\text {total }}(\mathrm{n} \rightarrow \mathrm{m})}{\longrightarrow} \quad \mathrm{CHOL}_{(\mathrm{m})}^{\text {(charged })} \\
& \mathrm{G}_{\mathrm{elec}}^{\mathrm{TI}}(\mathrm{n}) \text { 个 } \\
& \mathrm{CHOL}_{(\mathrm{n})}^{\text {(uncharged) }} \underset{\Delta \mathrm{G}_{\mathrm{LJ}}^{\mathrm{ABF}}(\mathrm{n} \rightarrow \mathrm{m})}{\longrightarrow} \quad \mathrm{CHOL}_{(\mathrm{m})}^{\text {(uncharged) }} \\
& \Delta G_{\text {total }}(n \rightarrow m)=\Delta G_{L J}^{A B F}(n \rightarrow m)-G_{\text {elec }}^{T I}(n)+G_{\text {elec }}^{T I}(m) \\
& \Delta G_{\text {total }}(n \rightarrow m)=\Delta G_{L J}^{A B F}(n \rightarrow m)+\Delta G_{\text {elec }}^{T I}(n \rightarrow m)
\end{aligned}
$$

We assumed that the initial $(n)$ and final $(m)$ states are the CHOL molecule harmonically restrained in the middle of bulk water and different depths in the bilayer, respectively.

\subsection{Partial volume and area per lipid}

Determination of the partial area and volume per lipid in complex lipid mixtures by MD simulations has received considerable attention in recent years [27,108]. Atom-based tessellation 
methods have been widely applied to obtain the partial area per lipid in the perpendicular plane of bilayers composed of complex lipid mixtures. Unfortunately, standard tessellation analysis for molecular area calculations in lipid bilayers depends on an arbitrary choice of which normal coordinate position of the bilayer plane should be tessellated.

The methodological approach taken in this study was based on the Hofsäß method [24], which provides a useful relation between bilayer thickness and molecular volumes to obtain lipid partial areas in binary mixtures.

A straightforward strategy to split the total area per lipid $a$ into its components $a_{A}$ and $a_{B}$, assuming a binary mixture of lipids $\mathrm{A}$ and $\mathrm{B}$, is given by:

$$
a(x)=(1-x) a_{A}(x)+x a_{B}(x)
$$

where $x$ is the molar fraction of lipid B.

The Hofsäß method [24] can be used to split the total area per lipid into partial areas. Subsequently, Edholm and Nagle proposed a variation of this method to improve the analysis of the volumetric part [109]. The central premise of the Hofsäß method [24] is to use the mathematical relation between area and volume to define a standard thickness $h(x)$ for the lipid bilayer as follows:

$$
h(x)=\frac{V(x)-N_{w} V_{w}}{a(x)}
$$

where $V$ is the total volume of the simulation box, $N_{w}$ is the total number of water molecules, $V_{w}$ is the volume of a water molecule and $a$ is the total area. 
For the specific case of a binary lipid mixture of lipids A and B, the partial area for A and B as function of CHOL $x$ can be expressed as:

$$
h(x)=2 \frac{V_{A}(x)}{a_{A}(x)}=2 \frac{V_{B}(x)}{a_{B}(x)}
$$

For convenience, one can define a function $w(x)$ that represents the volume ratio between $V_{B}$ and $V_{A}$,

$$
a_{B}(x)=w(x) a_{A}(x)
$$

and then, one can write:

$$
a_{A}(x)=\frac{a(x)}{a_{A}(x)[1-x(1-w(x)]}
$$

The standard analysis of the partial area per lipid does not take account of the free volume related to the lipid packing in bilayers. The Voronoi tessellation [110] is especially well-suited to avoid this problem. In short, this technique consists of defining several cells surrounding a particular set of particles getting the space closest to them. Thus, we have therefore made use of Voronoi tessellation via Voro++ program [111] in LAMMPS to capture the effective volume $V_{A}$ and $V_{B}$ occupied by the lipids in the simulation box.

\subsection{Cholesterol tilt angle distribution}


The tilt angle distribution was obtained by the polar angle (given in degrees) between a vector joining specific beads in the CHOL and DPPC molecules and the outward vector normal to the bilayer within the range of $0^{\circ}$ to $180^{\circ}$ (see Section 4 in SI).

\subsection{Lipid lateral self-diffusion coefficient}

Lipid lateral self-diffusion coefficients were obtained from the center-of-mass displacement of DPPC and CHOL molecules in the two-dimensional space parallel to the bilayer. The mean square displacement (MSD) as function of time is given by

$$
M S D(t)=\frac{1}{N} \sum_{i=1}^{N}\left\langle\left|\vec{r}_{i}\left(t+t_{0}\right)-\vec{r}_{l}\left(t_{0}\right)\right|^{2}\right\rangle
$$

which is related to the self-diffusion coefficient $D$ by

$$
D=\lim _{t \rightarrow \infty} \frac{1}{4 N t} \sum_{i=1}^{N}\left\langle\left|\vec{r}_{i}\left(t+t_{0}\right)-\vec{r}_{i}\left(t_{0}\right)\right|^{2}\right\rangle
$$

where $N$ is the number of lipids, and $r_{i}\left(t_{0}\right)$ and $r_{i}\left(t+t_{0}\right)$ are the position vectors of the lipid $i$ center-of-mass in the bilayer plane at the starting measurement time $t_{0}$ and at the limit measurement time $t+t_{0}$, respectively. The bracket notation was satisfied with the linear fit of lipid MSD as a function of time in a normal (linear) diffusional regime. Any "drifting effect" in the center-of-mass of the analyzed lipid groups was removed before the displacement of each of 
their particles was calculated. The lipid diffusion average and its uncertainties were calculated by block averaging [101,102].

\subsection{Parametrization protocol and topology}

Initially, an appropriate topology for the CHOL molecule was defined. The CG mapping scheme for the CT3-Me2b CHOL model designed by Daily and coworkers [84] for the MARTINI-FF [36] was chosen. Figure 1 shows the molecular structures of CHOL at different levels of resolution.

Figure 1. Molecular structures of $\mathrm{CHOL}$ at the $\mathrm{AA}$ and $\mathrm{CG}$ resolutions. (a) AA model of the CHARMM36-based CHOL molecule (74 atoms) in CPK representation. Atom colors are as follows: hydrogen (white), carbon (brown) and oxygen (red). (b) CG model of the CHOL molecule (9 beads) in VDW representation, and (c) its numbered two-dimensional projection. Bead colors are as follows: hydroxyl group (red), and ring/tail (brown).

(a)

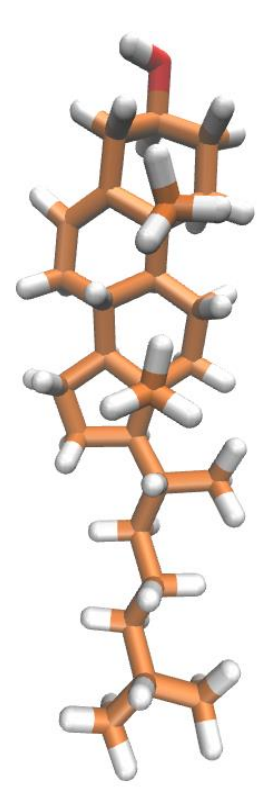

(b)

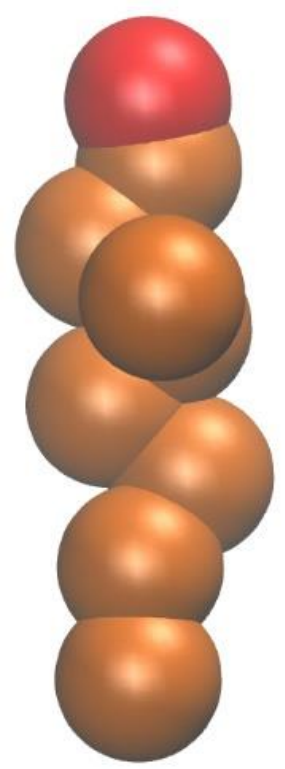

(c)

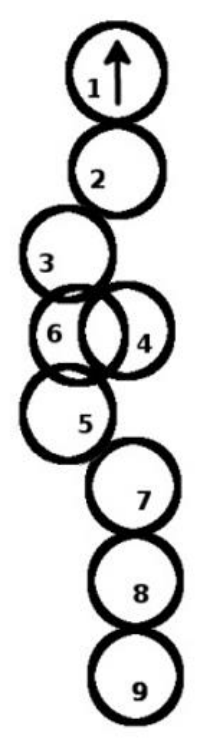


In particular, the pseudo-ring portion of the $\mathrm{CHOL}$ model was treated as a rigid body (Figure 1, beads 1 to 5). In this case, such an approximation is possible due to small distortions in the rigid $\mathrm{CHOL}$ ring structure. Therefore, numerical instabilities in the time propagation of motion equations were avoided by eliminating high frequency vibrational modes and improper dihedral potentials after the equilibration phase.

The parameterization protocol is similar to that used in MARTINI-FF [36]. Electrostatic and non-polar components of solvation free energies obtained from CHARMM36-FF [94,95]. MD simulations were used to set the Lennard-Jones (LJ) epsilon parameters of CHOL-water and CHOL-DPPC cross-interactions.

Regarding the other LJ parameters, default values from the ELBA-FF [37] for the ester and non-polar chemical blocks were used as starting point to describe both the hydroxyl-like and the non-polar CHOL beads, respectively. Similarly, the dipole moment present in the hydroxyl-like bead was refined by comparison with electrostatic free energy results from CHARMM36-FF [94,95] MD simulations (Table 1).

Parameterization of DPPC-CHOL cross-interactions and CHOL dipole adjustment considered simulation data from free energy calculations of CHOL partitioning in pure DPPC bilayer. Furthermore, CHOL-CHOL cross-interactions were adjusted on the basis of biophysical properties of high-cholesterol DPPC/CHOL (50:50 mol\%) bilayer simulations. In addition, intermediate $\mathrm{CHOL}$ content values were predicted by using the optimized set of parameters that best described the structural and energetic properties of both systems cited above.

\subsubsection{Optimizing CHOL model LJ cross-interactions}


Kessel and co-workers [112] emphasized that a single CHOL molecule desolvation free energy and specific location within the membrane are mainly driven by non-polar free energy contributions from DPPC-CHOL interactions. The authors also pointed out that $\mathrm{CHOL}$ distribution within a bilayer is determined by the elastic response of their neighboring lipids. In addition, some authors such as Jo and Khelashvili $[63,113]$ argued that CHOL orientational entropy as well as CHOL-CHOL and CHOL-phospholipid interactions play a crucial role in the free energy associated with the CHOL tilt modulus in heterogeneous lipid environments.

Here, particular attention was paid to calibrating DPPC-CHOL and CHOL-CHOL crossinteractions due to their direct relation with $\mathrm{CHOL}$ behavior within membranes. To optimize CHOL-CHOL and DPPC-CHOL cross-interactions, the original set of parameters for the polar and non-polar LJ sites available in ELBA-FF [37] were used as starting point. Default ELBA-FF [37] parameters were maintained for cross-interactions between ELBA water and polar and nonpolar DPPC and CHOL ELBA CG sites during parameterization.

First, the potential of the mean force (PMF) related to the free energy that was necessary to transfer an "uncharged" CHOL molecule from bulk water to the middle of the DPPC bilayer was obtained via CHARMM36-FF and ELBA-FF MD simulations. LJ epsilon values of CHOLDPPC cross-interactions were scaled up by multiplicative factors $(1.0,1.05,1.10,1.15$ and 1.20) over default parameter values used in ELBA-FF (the effect over PMFs can be viewed in Figure 2). Therefore, the cross-interaction between CHOL and DPPC was adjusted by seeking agreement between the AA and CG profiles.

Figure 2. Potential of mean force for a "non-charged" CHOL molecule, related to the reversible work necessary for its transfer from bulk water to the middle of the DPPC bilayer. The free energy 
profile results are shown by different colors for each scaled cross-interaction between CHOL and DPPC molecules. The black line represents the reference profile obtained by all-atom simulations using CHARMM36-FF. The orange, red, magenta, indigo, and green colors represent the scaled epsilon cross-interaction values by 1.0, 1.05, 1.10, 1.15, and 1.20 factors, respectively. Error bars have been omitted for the sake of clarity.



Similarly, the CHOL-CHOL self-interaction was also adjusted by employing multiplicative factors (in this case 1.0, 1.05, 1.10, and 1.15) over the default set of ELBA-FF 
parameters. Our aim was to choose the set of parameters that best described CHOL tilts and the partial area per CHOL and DPPC as compared to atomistic reference data.

\subsubsection{Optimizing CHOL model dipole moment}

The magnitude of the dipole moment embedded in bead 1 (see Figure 1, item a) was fine-tuned to match the reference value found for the total free energy of the CHARMM36 CHOL [94,95] partitioning from bulk water into the hydrocarbon core of the DPPC bilayer [96]. The optimal dipole (4.92 D) was obtained by scaling up the dipole vector that describes the ester group (2.00 D) in ELBA-FF [37] (for more details, see Section 2 in SI).

Table 1 summarizes AA and CG predictions for the Electrostatic and LJ free energy contributions for moving the CHOL molecule from bulk water to the middle of the DPPC bilayer. Table 1 also provides a comparison between the CHOL model predictions and those obtained from the GROMOS87, CAVS, and MARTINI FFs [85,114] for the total free energy of CHOL partitioning in pure DPPC bilayers.

Table 1. Electrostatic and LJ contributions for the total free energy of CHOL partitioning in pure DPPC bilayer calculated by ELBA-FF [37] and CHARMM36-FF $[94,95] . G_{\text {elec }}^{T I}(w)$ and $G_{\text {elec }}^{T I}(c)$ are the absolute reversible electrostatic work to charge one CHOL molecule harmonically restrained in both bulk water and pure DPPC bilayer center of masses, respectively. $\Delta G_{\text {elec }}^{T I}(\mathrm{w} \rightarrow$ c) and $\triangle G_{L J}^{A B F}(\mathrm{w} \rightarrow \mathrm{c})$ represents the Electrostatic and LJ free energy difference, respectively, necessary to transfer the CHOL molecule from bulk water to the bilayer center. $\triangle G_{\text {total }}^{D P P C}(\mathrm{w} \rightarrow \mathrm{c})$ denotes the total free energy cost for moving CHOL from bulk water to the bilayer center. 


\begin{tabular}{|c|c|c|c|c|c|}
\hline Force Field & $G_{\text {elec }}^{T I}(\mathrm{w})$ & $G_{\text {elec }}^{T I}(c)$ & $\Delta G_{\text {elec }}^{T I}(\mathrm{w} \rightarrow \mathrm{c})$ & $\Delta G_{L J}^{A B F}(\mathrm{w} \rightarrow \mathrm{c})$ & $\Delta G_{\text {center }}^{D P P C}(\mathrm{w} \rightarrow \mathrm{c})$ \\
\hline ELBA & $-8.0 \pm 0.2$ & $-0.1 \pm 0.4$ & $+7.9 \pm 0.5$ & $-20.2 \pm 4.0$ & $-12.3 \pm 4.0$ \\
\hline CHARMM & $-8.0 \pm 0.2$ & $-0.2 \pm 0.1$ & $+7.8 \pm 0.2$ & $-20.1 \pm 3.6$ & $-12.3 \pm 3.6$ \\
\hline GROMOS & - & - & - & - & $-13.4[114]$ \\
\hline CAVS & - & - & - & - & $-15.5[85]$ \\
\hline MARTINI & - & - & - & - & $-17.6[114]$ \\
\hline
\end{tabular}

\section{RESULTS AND DISCUSSION}

\subsection{Structural properties}

A characteristic effect of the presence of CHOL in bilayers consisting of mixed lipids is the socalled "cholesterol condensing effect", characterized by a sharp decrease in the phospholipid bilayer area (and free volume). Theoretical models such as the Superlattice Model $[115,116]$ and the Umbrella Model $[117,118]$ were proposed to explain such effect. The Superlattice Model successfully predicts highly regular distributions by symmetrical geometric assumptions of the system, as confirmed by experimental techniques [119-121]. This model also suggests a link with the long-range repulsive interaction owing to the cross-sectional area difference between sterol and lipids. In turn, the Umbrella Model postulates that the shape complementarity of phospholipids "covering" the CHOL molecule avoids the free energy penalties of the CHOL molecules interacting with interfacial water in phospholipid bilayers. The latter model plays a crucial role in explaining the "cholesterol condensing effect" in phospholipids [117,118].

Regarding DPPC/CHOL mixtures, previous predictions obtained by mean-field calculations and MC/MD simulations demonstrated that the partial area per DPPC decreases with increasing CHOL content [122,123]. 
Figure 3 shows the total and partial area per DPPC and CHOL for different CHOL mole fractions. Area values drop in the case of partial molecular areas of the CHOL model with 0 mol\% to $50 \mathrm{~mol} \% \mathrm{CHOL}$ content in the DPPC/CHOL mixtures.

Figure 3. The total (diamond) and partial molecular area of DPPC (triangle) and CHOL (square) as a function of CHOL content (from $0 \mathrm{~mol} \%$ to $50 \mathrm{~mol} \%$ ) for DPPC/CHOL mixtures. The results of the DPPC/CHOL model are shown in black, and the data from atomistic simulations are shown in blue [109] and green [108] colors, respectively. The full (total area per lipid), dotted (partial area per DPPC), and dashed (partial area per CHOL) lines in the figure are drawn to guide the eye.

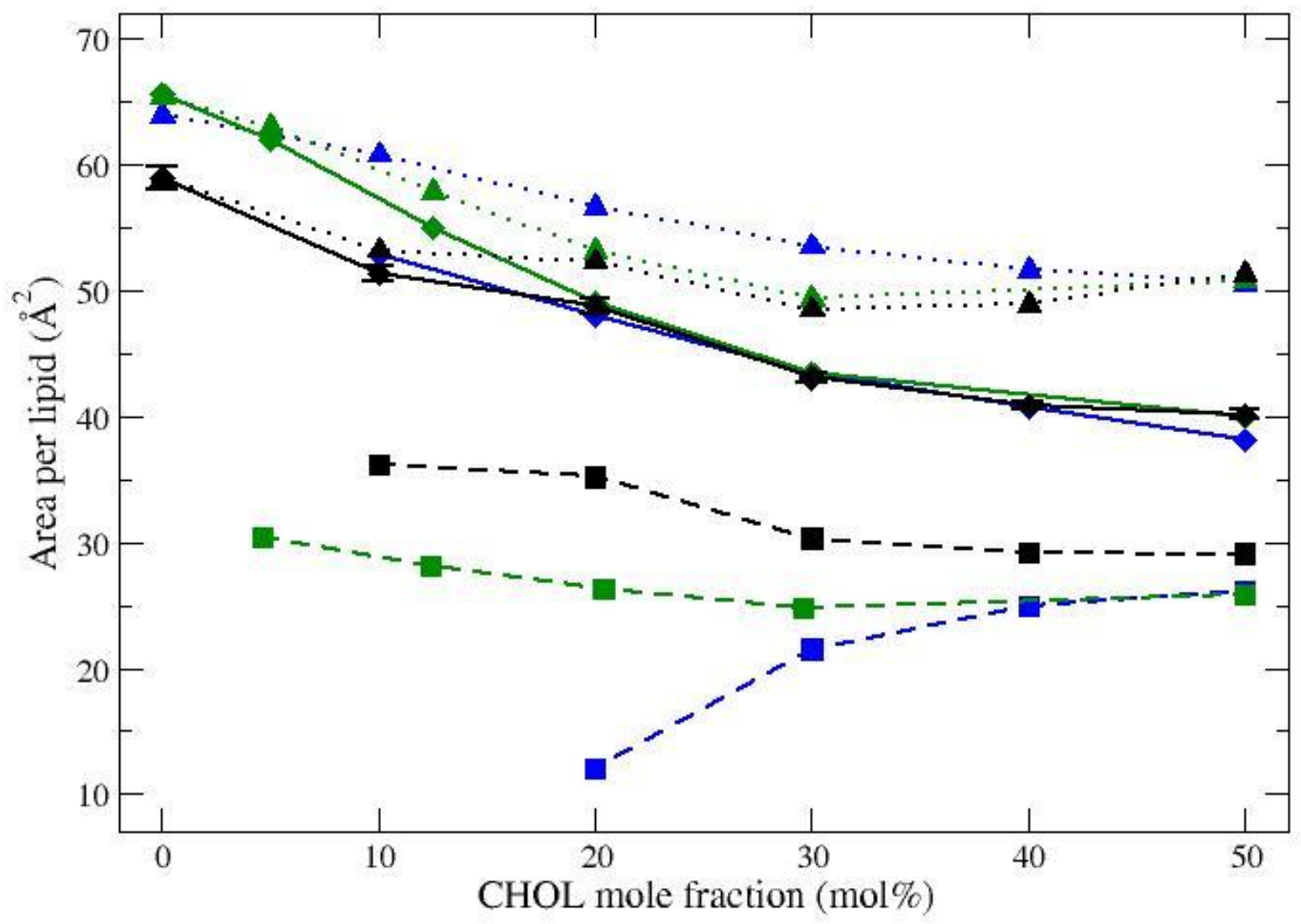


Hofsäß and coworkers [24] and Edholm and coworkers [109] had already reported that CHOL addition to DPPC affects the partial CHOL area: areas decrease from 29 to $27 \AA^{2}$ and from 28 to $23 \AA^{2}$, respectively. Furthermore, Chiu and coworkers [23] found partial area per CHOL equal to $22.3 \AA^{2}$ in high-cholesterol regime. The discrepancy between the AA predictions for the partial CHOL area (Figure 3) results from their different forms to incorporate the local perturbation of $\mathrm{CHOL}$ in the partial area calculations.

In general, our CG DPPC/CHOL simulations correctly describe the reduction in the partial and total area per CHOL and DPPC lipids within the range of $0 \mathrm{~mol} \%$ to $50 \mathrm{~mol} \% \mathrm{CHOL}$. For the DPPC/CHOL mixture in the range of $0 \mathrm{~mol} \%$ to $30 \mathrm{~mol} \%$, the partial area per DPPC decreases significantly, being almost constant for CHOL contents greater than $30 \mathrm{~mol} \%$. Such reductions in the partial lipid area and the consequent contraction in the bilayer plane correspond to the aforementioned "condensing effect of cholesterol" concept, which has been widely discussed in the literature. [124-130].

Concerning the area per lipid of the pure DPPC bilayer, experimental data have suggested a value equal to $62.9 \AA^{2}$ [131], whereas recent atomistic simulations have inferred values ranging from 59.1 to $62.9 \AA^{2}[94,132,133]$. Our prediction for the pure DPPC-ELBA bilayer model is 58.9 $\AA^{2}$.

Table 2 highlights the average values of tilt angles $\theta$ (in degrees) and distances along the bilayer normal $\mathrm{D}_{\mathrm{Z}}$ (in $\AA$ ) for the $\mathrm{CHOL}$ molecules and DPPC hydrocarbon tails in DPPC/CHOL bilayers containing 40 and $50 \mathrm{~mol} \% \mathrm{CHOL}$, respectively. The first parameter is closely related to CHOL ordering abilities and orientation within the bilayer. The latter parameter helps to validate the CHOL location normal to the bilayer. 
Table 2. Average values of tilt angles $(\theta)$ for CHOL molecules and DPPC hydrocarbon tails, and distances from bilayer center $\left(D_{Z}\right)$ to pseudo-atom groups of CHOL molecules in DPPC/CHOL bilayers at two different CHOL concentration (40 and 50 mol\% CHOL).

\begin{tabular}{|c|c|c|c|c|c|}
\hline \multicolumn{2}{|c|}{ Properties } & ELBA & Coarse-Grained & Atomistic & Experimental \\
\hline$\Theta_{\mathrm{CHOL}}$ & {$\left[{ }^{\circ}\right]$} & $\begin{array}{l}18.4 \pm 1.6 \dagger \\
17.7 \pm 1.3 \dagger\end{array}$ & $\begin{array}{c}9-10 \div[134] 15 \div[114] \\
21 \div[85]\end{array}$ & $\begin{array}{c}11-12.3+[26] 13+[114] \\
10.5+[135]\end{array}$ & $16 \div[136]$ \\
\hline$\Theta_{\text {DPPC }}$ & {$\left[{ }^{\circ}\right]$} & $\begin{array}{l}20.3 \pm 2.2 \dagger \\
24.2 \pm 2.3 \dagger\end{array}$ & - & $20.3+[24]$ & $16-25[136]$ \\
\hline $\mathrm{D}_{\mathrm{Z}}{ }^{\mathrm{CHOL}}$ & {$[\AA]$} & $\begin{array}{l}11.6 \pm 0.2 \dagger \\
11.3 \pm 0.1 \dagger\end{array}$ & $17.0 \dagger[114] \quad 20.0 \dagger \quad[85]$ & $13.2 \div[135] 18.0 \div[114]$ & $12.9 \div[137]$ \\
\hline $\mathrm{D}_{\mathrm{Z}}^{\mathrm{OH}}$ & {$[\AA]$} & $\begin{array}{l}16.8 \pm 0.2 \dagger \\
16.6 \pm 0.2 \dagger\end{array}$ & $16.9 \div[134]$ & $18.2-18.4 \div[26]$ & - \\
\hline $\mathrm{D}_{\mathrm{Z}}^{\mathrm{RING}}$ & {$[\AA]$} & $\begin{array}{l}11.7 \pm 0.2 \dagger \\
11.4 \pm 0.2 \dagger\end{array}$ & - & - & - \\
\hline $\mathrm{D}_{\mathrm{Z}}^{\mathrm{TAIL}}$ & {$[\AA]$} & $\begin{array}{l}4.4 \pm 0.2 \dagger \\
4.1 \pm 0.1 \dagger\end{array}$ & - & $4.0 \dagger[135]$ & - \\
\hline
\end{tabular}

$\dagger 40 \mathrm{~mol} \% \mathrm{CHOL}$

$\$ 50 \mathrm{~mol} \% \mathrm{CHOL}$

The average distance from CHOL molecules and CHOL-hydroxyl $(\mathrm{OH})$ groups are, respectively, $11.6 \AA$ and $16.8 \AA$ to the bilayer center in the DPPC/CHOL (60:40 mol\%) system. These results are consistent with data acquired by experimental and simulation studies of binary DPPC/cholesterol bilayers containing equivalent CHOL amount [135,137].

The CHOL model reasonably predicts the tilt angle for CHOL molecules in DPPC bilayers with 40 and $50 \mathrm{~mol} \% \mathrm{CHOL}$ content, with average values equal to $18.4^{\circ}$ and $17.7^{\circ}$, respectively. Experimental prediction for CHOL tilts in the DPPC/CHOL mixture with CHOL at $40 \mathrm{~mol} \%$ has afforded an average value equal to $16^{\circ}[136]$. The CHOL model has been shown slightly higher 
tilt average values as compared to values obtained by AA simulations. Furthermore, DPPC hydrocarbon chains in DPPC/CHOL mixtures containing $40 \mathrm{~mol} \% \mathrm{CHOL}$ has shown an average value equal to $20.3^{\circ}$, which well agrees with AA predictions [136].

We also compared experimental volumetric data for pure and hydrated $\mathrm{CHOL}$ between the CHOL model and equivalent data from literature. Renshaw and coworkers [138] observed two values of molecular CHOL volume in bulk water: $627 \AA^{3}$ and $606 \AA^{3}$ for the anhydrous and monohydrated states, respectively. Additionally, an experimental bulk density equal to $1.1 \mathrm{~g} / \mathrm{cm}^{3}$ has been found for pure $\mathrm{CHOL}$ at $298.15 \mathrm{~K}$. Our simulation predictions for the hydrated $\mathrm{CHOL}$ molecular volume and for the bulk CHOL density are $613.1 \AA^{3}$ and $1.2 \mathrm{~g} / \mathrm{cm}^{3}$, respectively. These results are in line with the results cited above and contribute to validating our parameterization.

\subsection{Dynamic properties}

The dynamic properties of membrane lipids are intrinsically related to lipid distribution in cells, flip-flop diffusion, lipid raft formation, and membrane permeability.

The free-volume model [139] assumes that lateral lipid diffusion in bilayers is a result of the random occurrence of free volume portions of which a lipid in its vicinity takes place. On the other hand, Falck and co-workers [140] postulate that lateral lipid diffusion is a continuous and correlated motion of lipids.

Particularly, the free volume theory successfully explains why lipid diffusion decreases with increasing CHOL content [141]. However, Falck and co-workers argue that more realistic models should be used to predict lipid diffusion in lipid bilayers quantitatively [108]. Subsequently, Almeida and coworkers [142] clarified the validity of the free volume model to describe experimental data for lipid diffusion in PC/CHOL mixtures quantitatively. 
CHOL influence on lateral lipid diffusion is well established in the literature. Countless experimental and theoretical studies on self-diffusion in lipid membrane mixtures containing CHOL have been published [21,24,108,141,143-157].

Scherfeld and co-workers [152] verified an increase in the lipid diffusion rates for CHOL contents ranging from $33 \mathrm{~mol} \%$ to $67 \mathrm{~mol} \%$ in Giant Unilamellar Vesicles (GUVs) of DPPC/CHOL mixture by Fluorescence Correlation Spectroscopy (FCS). Several experimental results corroborated that the lateral PC lipid self-diffusion coefficient diminishes upon increasing CHOL content $[143,147,148,150]$. Moreover, Kuo and co-workers [158] noticed that less than 10 mol $\%$ CHOL content accelerates lipid diffusion in DPPC multilayers by a pulsed gradient proton Nuclear Magnetic Resonance (NMR) spin-echo study. The same authors found that the diffusion coefficient decreases in high CHOL concentrations. On the basis of the pulsed field gradient NMR technique, Filippov and co-workers [151] inferred a similar increase in lateral diffusion at small CHOL content in the DOPC/CHOL system. They also observed that lipid diffusion decreases substantially when CHOL concentration increases from $5 \mathrm{~mol} \%$ to $15 \mathrm{~mol} \%$ but remains constant thereafter.

Experimental methods like pulsed NMR and FRAP work in the millisecond timescale, whereas contemporary MD simulations usually do not reach such long timescale. Thus, direct comparison of experimental and simulation results concerning lipid diffusion should take this into account.

Most MD simulation studies agree that lipid diffusion coefficients decrease with increasing CHOL content in PC/CHOL mixtures [24,108,159].

Remarkably, our CG simulation results (Figure 6) correctly describe most of the experimental observations cited above. Compared to CHOL, the DPPC diffusion rate is slightly 
lower for $\mathrm{CHOL}$ concentrations up to $30 \mathrm{~mol} \%$. As for $\mathrm{CHOL}$ concentrations greater than 30 mol\%, the DPPC and CHOL diffusion rates are not significantly different. In addition, our DPPC/CHOL model at low CHOL content come up with diffusion rates around an order of magnitude faster than those found at CHOL-rich regime, whereas there is no significant difference in MARTINI predictions $[74,160]$.

Figure 6. Lateral self-diffusion coefficient for DPPC and CHOL as a function of different $\mathrm{CHOL}$ concentrations. The CHOL and DPPC diffusion rates are shown by the triangle and square symbols, respectively. The CHOL and DPPC diffusion rate predictions are shown in black, and the data derived from atomistic simulations are indicated in blue [108] and green [24] colors, respectively. For the sake of clarity, the upper inset shows the CHOL and DPPC diffusion rates on a logarithmic scale as function of $\mathrm{CHOL}$ concentration. 


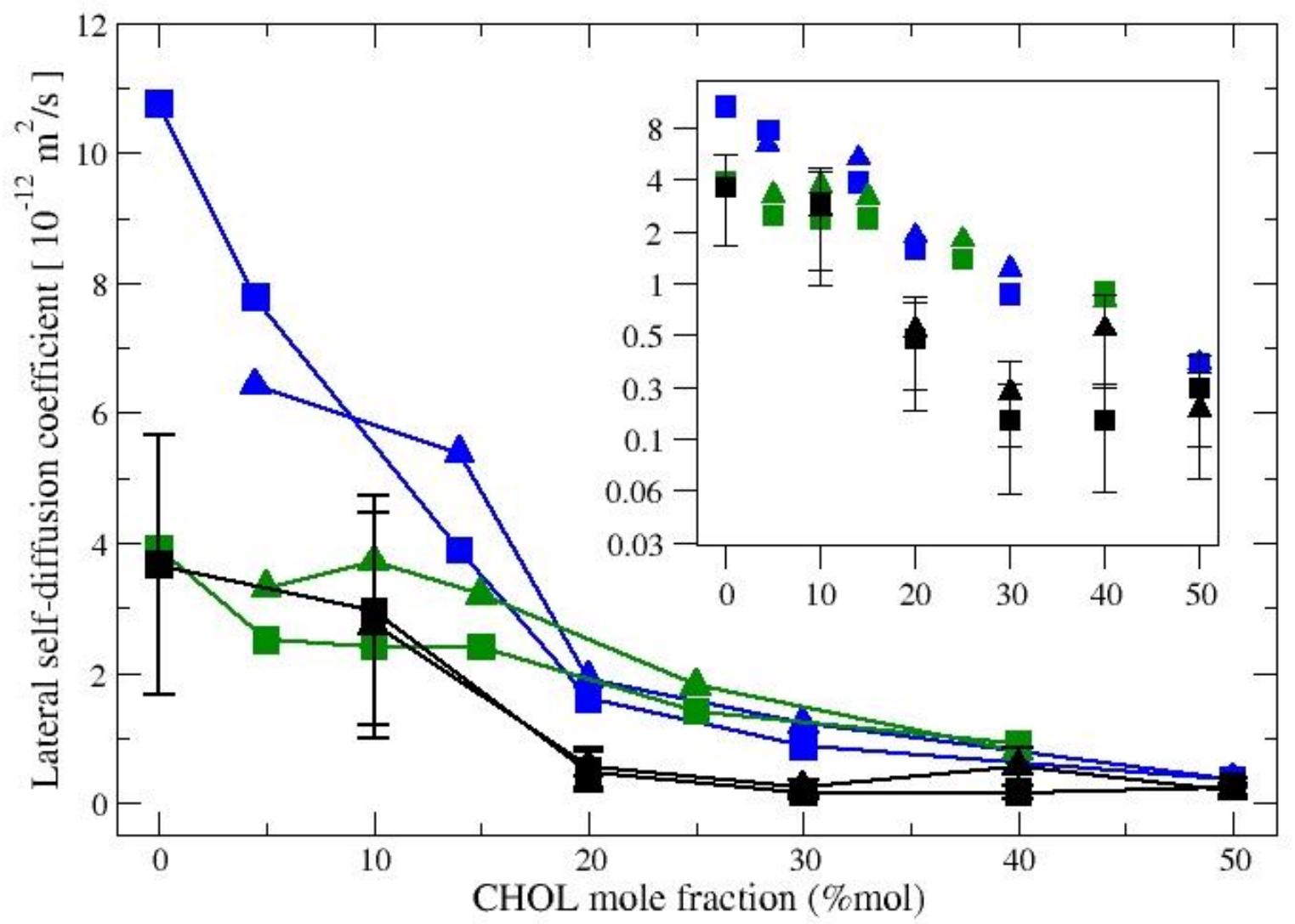

The fact that CHOL and DPPC have similar diffusion rates at high CHOL content can be interpreted on the basis of the hypothesis of Hofsäß and co-workers [24], who argued that DPPC molecules hold on to almost the same vicinity of CHOL molecules along time, thereby leading to mutual diffusion between them.

\subsection{Thermodynamic properties}

Free energy calculations via MD simulations have been employed to understand how $\mathrm{CHOL}$ affects organic solute transport through bilayers. Jedlovszky and Mezei [161] explored how CHOL impacts the transport of small solutes through the membrane, to observe that CHOL hydroxyl 
groups are located within the membrane plays a pivotal role in the solvation free energy of polar and apolar solutes.

Despite the vast literature on CHOL modulation of biophysical properties and small solute transport in biomembranes, few studies have provided a quantitative picture of the thermodynamic process involved in CHOL partitioning in biomembranes.

Jo and co-workers [113] suggested that an unfavorable enthalpic component due to CHOL (hydroxyl) polar group desolvation is balanced by a favorable entropic factor arising from enhanced lipid dynamics promoted by CHOL. Moreover, Bennett and coworkers [114] found a total free energy for moving a single $\mathrm{CHOL}$ molecule from bulk water to its equilibrium position in DPPC/CHOL bilayers (60/40 mol\%) equal to $-21.3 \mathrm{kcal} / \mathrm{mol}$ and $-20.6 \mathrm{kcal} / \mathrm{mol}$ when they used AA (GROMOS87-FF) and CG (MARTINI-FF) models, respectively. These authors also found a favorable free energy difference for the CHOL partitioning process in the middle of DPPC/CHOL bilayers $(60 / 40 \mathrm{~mol} \%)$ - around $-12.7 \mathrm{kcal} / \mathrm{mol}$ and $-17.0 \mathrm{kcal} / \mathrm{mol}$ - by using the GROMOS87FF and MARTINI-FF, respectively. Additionally, the PMF calculated by Díaz-Tejada and coworkers [80] for lateral CHOL partitioning from a pure DPPC moiety to another moiety containing DPPC at $50 \%$ CHOL content gave a total free energy difference of around $3.6 \mathrm{kcal} / \mathrm{mol}$.

To calculate the total free energy of CHOL partitioning in DPPC with CHOL at $40 \mathrm{~mol} \%$ content, we first estimate the Electrostatic and LJ free energy difference of CHOL desolvation at its equilibrium position in the bilayer, to achieve $\Delta G_{\text {elec }}^{T l}(w \rightarrow e q)=-0.8 \pm 0.4 \mathrm{kcal} / \mathrm{mol}$ and $\Delta G_{L J}^{A B F}(w \rightarrow e q)=-21.4 \pm 2.3 \mathrm{kcal} / \mathrm{mol}$, respectively 
Figure 7. Permeation free energy profile for a "non-charged" CHOL molecule going from bulk water toward the middle of the DPPC/CHOL (60:40 mol\%) bilayer. The vertical dashed lines delimit the main regions of the membrane. Vertical double-headed arrows depict the LJ free energy difference for moving CHOL between different regions in the bilayer system, and vertical dotted lines delimit the main regions of the membrane based on the numerical density results (see Figure S17 in SI)

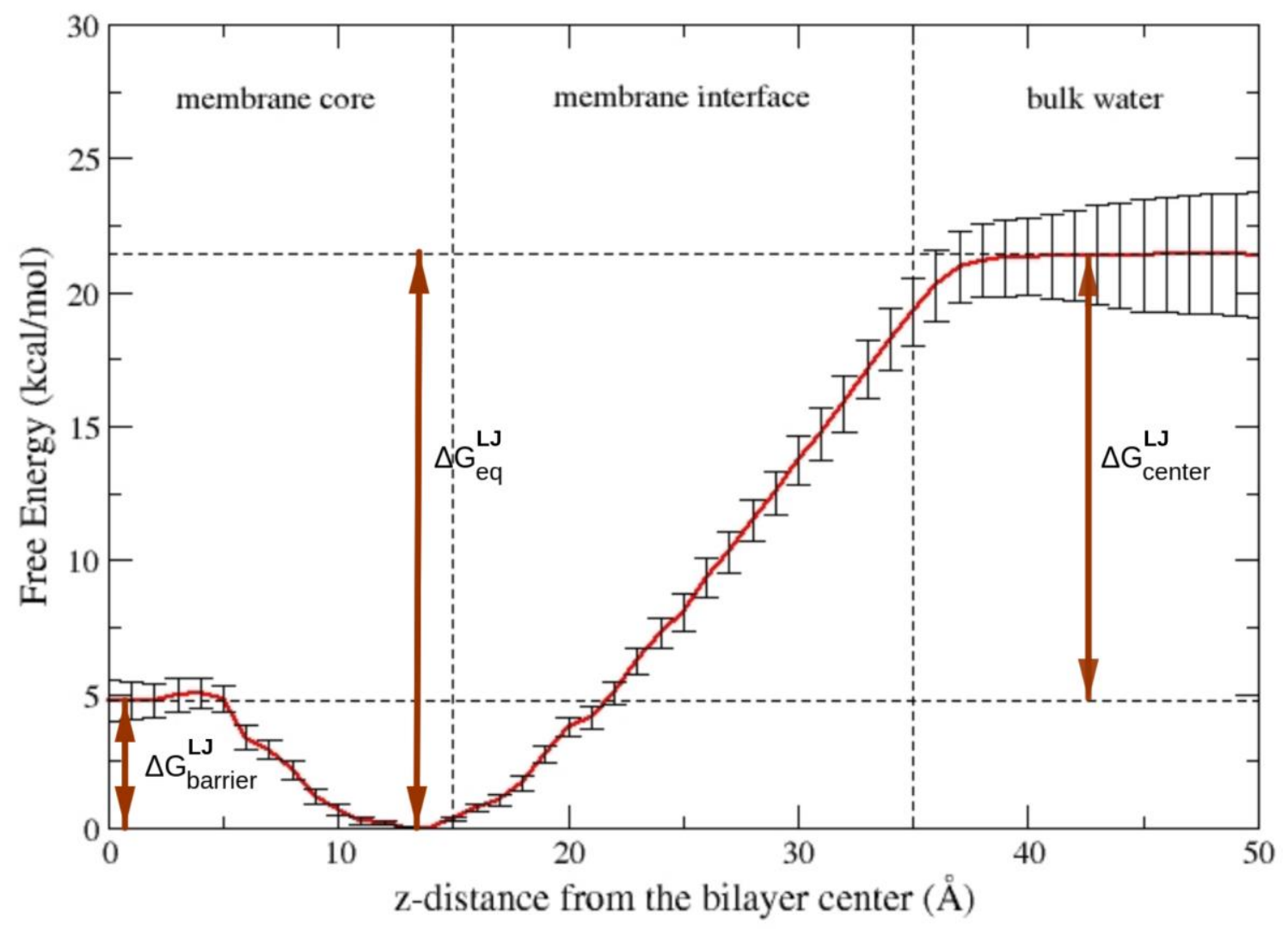

Table 3 details the free energy results by comparing different FF predictions for the total free energy of CHOL partitioning in membranes. It is noticed a favorable total free energy difference for moving the CHOL molecule from bulk water to its equilibrium position in the DPPC/CHOL $(60: 40 \mathrm{~mol} \%)$ bilayer, with $\Delta G_{e q}^{D P C H}(w \rightarrow e q)=-22.2 \pm 2.4 \mathrm{kcal} / \mathrm{mol}$. These results agree fairly well with previous atomistic and coarse-grained simulations $[85,114]$. The main 
contribution to the overall free energy of CHOL membrane partitioning comes from the non-polar component. Such results corroborate previous theoretical predictions for a similar thermodynamic process of CHOL partitioning in membranes [112].

Table 3. (a) Comparison of the total free energy of CHOL partitioning in DPPC bilayer at 40 mol\% CHOL content according to ELBA-based, GROMOS-based, MARTINI-based, and CAVSbased simulations [85,114]. (b) Schematic representation of the CHOL partitioning from aqueous phase to different moieties in the bilayer. $\Delta G_{e q}^{D P C H}(w \rightarrow e q)$ means the total free energy of CHOL desolvation at its equilibrium position in the bilayer, $\Delta G_{c e n t e r}^{D P C H}(w \rightarrow c)$ represents the total free energy of CHOL desolvation at the bilayer center, and $\Delta G_{\text {barrier }}^{D P C H}(e q \rightarrow c)$ denotes the free energy cost to move the CHOL molecule from its equilibrium position to the bilayer center.

(a)

\begin{tabular}{|c|c|c|c|c|}
\hline Free Energy $(\mathrm{kcal} / \mathrm{mol})$ & ELBA & GROMOS & MARTINI & CAVS \\
\hline$\Delta G_{\text {eq }}^{D P C H}(w \rightarrow e q)$ & $-22.2 \pm 2.4$ & -21.3 & -20.6 & -22.7 \\
\hline$\Delta G_{\text {center }}^{D P C H}(w \rightarrow c)$ & $-12.0 \pm 2.5$ & -12.7 & -17.0 & -18.2 \\
\hline$\Delta G_{\text {barrier }}^{D P C H}(e q \rightarrow c)$ & $+10.0 \pm 1.0$ & +9.8 & +6.0 & +6.5 \\
\hline
\end{tabular}

(b)

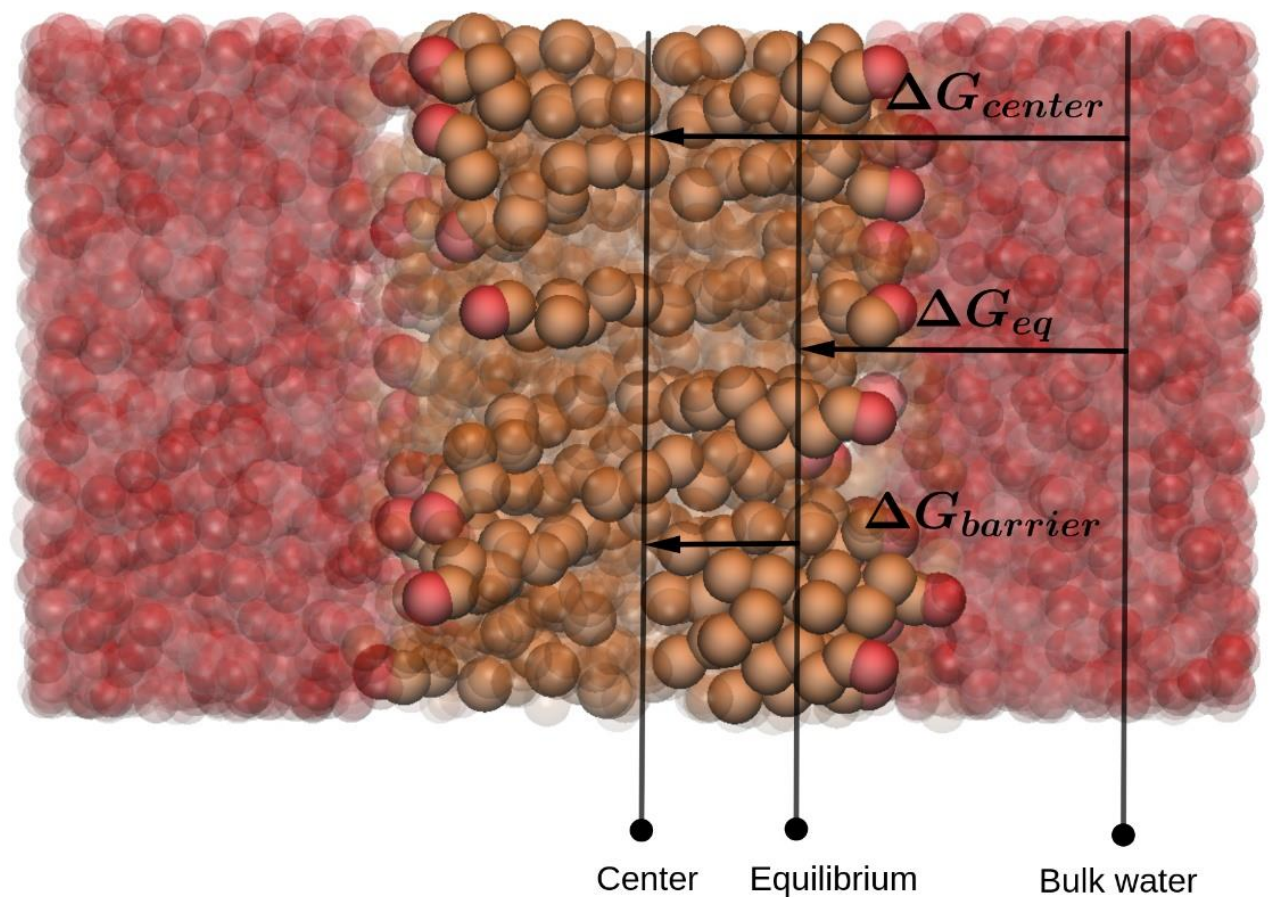




\section{CONCLUSION}

This paper presents a new cholesterol model (CHOL) for the electrostatic-based ELBA force field. We have parameterized and validated the CHOL model on the basis of energetic and structural properties of experimental and atomistic reference data. Such set of parameters were employed to predict how the CHOL concentration (from $0 \mathrm{~mol} \%$ to $50 \mathrm{~mol} \%$ ) affects the hydrated DPPC bilayer model.

In general, the CHOL model satisfactorily agrees with data derived from atomistic simulations and experiments in relation to the hydrated CHOL molecular volume, pure CHOL bulk density, CHOL self-diffusion and partial molecular area and volume in the tested binary DPPC/CHOL mixtures.

The CHOL model provides a fair prediction for the free energy of CHOL partitioning in membranes consisting of pure and cholesterol-rich DPPC bilayers as compared to previous literature simulation data.

\section{AUTHOR INFORMATION}

Corresponding Author

*E-mail: siani@usp.br

\section{ACKNOWLEDGMENTS}

PS thanks CNPq for a doctoral fellowship. Computations were carried out at USP HighPerformance Computing (USP-HPC), on the Danish e-Infrastructure Cooperation (DeIC) supercomputer ABACUS 2.0, and local SGI cluster premises supported by FAPESP grant (Process 
2013/08166-5). This work is supported by FAPESP grant (Process 2017/03204-7). HK is supported by Lundbeckfonden.

\section{REFERENCES}

1. Silvius JR (2003) Biochimica et Biophysica Acta (BBA)-Biomembranes 1610(2):174

2. Lawrence JC, Saslowsky DE, Edwardson JM, Henderson RM (2003) Biophysical journal 84(3):1827

3. Marsh D (2009) Biochimica et Biophysica Acta (BBA)-Biomembranes 1788(10):2114

4. Cecchi C, Nichino D, Zampagni M, Bernacchioni C, Evangelisti E, Pensalfini A, Liguri G, Gliozzi A, Stefani M, Relini A (2009) Biochimica et Biophysica Acta (BBA)-Biomembranes 1788(10):2204

5. London E (2004) Journal of Biological Chemistry 279(11):9997

6. Zhuang L, Kim J, Adam RM, Solomon KR, Freeman MR (2005) Journal of Clinical Investigation 115(4):959

7. Simons K, Ikonen E (1997) nature 387(6633):569

8. De Almeida RF, Fedorov A, Prieto M (2003) Biophysical journal 85(4):2406

9. London E, Brown DA (2000) Biochimica et Biophysica Acta (BBA)-Biomembranes 1508(1):182

10. Brown DA, London E (2000) Journal of Biological Chemistry 275(23):17221

11. McMullen TP, Lewis RN, McElhaney RN (2004) Current opinion in colloid \& interface science 8(6):459

12. Hooper NM (1999) Molecular membrane biology 16(2):145

13. Papahadjopoulos D, Cowden M, Kimelberg H (1973) Biochimica et Biophysica Acta (BBA)Biomembranes 330(1):8

14. Szabo $G(1974)$ Nature $252: 47$

15. Grunze M, Deuticke B (1974) Biochimica et Biophysica Acta (BBA)-Biomembranes 356(1):125

16. Pitman MC, Grossfield A, Suits F, Feller SE (2005) Journal of the American Chemical Society 127(13):4576

17. Pownall HJ, Massey JB, Kusserow SK, Gotto Jr AM (1979) Biochemistry 18(4):574

18. Kleemann W, McConnell HM (1976) Biochimica et Biophysica Acta (BBA)-Biomembranes 419(2):206

19. Wood WG, Eckert GP, Igbavboa U, Müller WE (2003) Biochimica et Biophysica Acta (BBA)Biomembranes 1610(2):281

20. Frédérick J-M, Rodgers JM, Willems TF, Smit B (2010) Biophysical journal 99(11):3629

21. Chiu S, Jakobsson E, Scott HL (2001) Biophysical journal 80(3):1104

22. Khelashvili GA, Scott $H$ (2004) The Journal of chemical physics 120(20):9841

23. Chiu S, Jakobsson E, Mashl RJ, Scott HL (2002) Biophysical Journal 83(4):1842

24. Hofsäß C, Lindahl E, Edholm O (2003) Biophysical journal 84(4):2192

25. Kučerka N, Perlmutter JD, Pan J, Tristram-Nagle S, Katsaras J, Sachs JN (2008) Biophysical journal 95(6):2792

26. Smondyrev AM, Berkowitz ML (1999) Biophysical Journal 77(4):2075

27. Pandit SA, Vasudevan S, Chiu S, Mashl RJ, Jakobsson E, Scott H (2004) Biophysical journal 87(2):1092

28. Pasenkiewicz-Gierula M, Róg T, Kitamura K, Kusumi A (2000) Biophysical journal 78(3):1376 
29. Scott H (1991) Biophysical journal 59(2):445

30. Berkowitz ML (2009) Biochimica et Biophysica Acta (BBA)-Biomembranes 1788(1):86

31. Miao L, Nielsen M, Thewalt J, Ipsen JH, Bloom M, Zuckermann MJ, Mouritsen OG (2002) Biophysical journal 82(3):1429

32. Ferreira TM, Coreta-Gomes F, Ollila OS, Moreno MJ, Vaz WL, Topgaard D (2013) Physical Chemistry Chemical Physics 15(6):1976

33. Róg T, Pasenkiewicz-Gierula M (2001) Biophysical journal 81(4):2190

34. de Meyer F, Smit B (2009) Proceedings of the National Academy of Sciences 106(10):3654

35. Frazier ML, Wright JR, Pokorny A, Almeida PF (2007) Biophysical journal 92(7):2422

36. Marrink SJ, Risselada HJ, Yefimov S, Tieleman DP, De Vries AH (2007) The journal of physical chemistry B 111(27):7812

37. Orsi M, Essex JW (2011) PloS one 6(12):e28637

38. Darré L, Machado MR, Brandner AF, González HC, Ferreira Sn, Pantano S (2015) Journal of chemical theory and computation 11(2):723

39. Uusitalo J, Ingólfsson HI, Akhshi P, Tieleman DP, Poolman B, Herrmann A, Marrink SJ (2014) Biophysical Journal 106(2):803a

40. Uusitalo JJ, Ingólfsson HI, Akhshi P, Tieleman DP, Marrink SJ (2015) Journal of chemical theory and computation 11(8):3932

41. Schmalhorst PS, Deluweit F, Scherrers R, Heisenberg C-P, Sikora M (2017) Journal of Chemical Theory and Computation

42. Nawaz S, Carbone P (2014) The Journal of Physical Chemistry B 118(6):1648

43. Ndao M, Devémy J, Ghoufi A, Malfreyt $P$ (2015) Journal of chemical theory and computation 11(8):3818

44. Siani P, de Souza R, Dias L, Itri R, Khandelia H (2016) Biochimica et Biophysica Acta (BBA)Biomembranes 1858(10):2498

45. Uusitalo JJ, Ingólfsson HI, Marrink S-J (2013) Biophysical Journal 104(2):169a

46. Orsi M (2014) Molecular Physics 112(11):1566

47. Orsi M (2014) Chemical and Biological Technologies in Agriculture 1(1):10

48. Orsi M, Ding W, Palaiokostas M (2014) Journal of chemical theory and computation 10(10):4684

49. Genheden S, Essex JW (2015) Journal of chemical theory and computation 11(10):4749

50. Genheden S (2017) Journal of Computer-Aided Molecular Design:1

51. Genheden S (2015) Journal of chemical theory and computation 12(1):297

52. Genheden S, Eriksson LA (2016) Journal of chemical theory and computation 12(9):4651

53. Marrink SJ, Tieleman DP (2013) Chemical Society Reviews 42(16):6801

54. Izvekov S, Voth GA (2005) The Journal of Physical Chemistry B 109(7):2469

55. Izvekov S, Voth GA (2005) The Journal of chemical physics 123(13):134105

56. Moore TC, lacovella CR, McCabe C (2014) The Journal of chemical physics 140(22):06B606_1

57. Reith D, Pütz M, Müller-Plathe F (2003) Journal of computational chemistry 24(13):1624

58. Dunn WL (1981) Journal of Computational Physics 41(1):154

59. Ipsen JH, Karlström G, Mourtisen O, Wennerström H, Zuckermann M (1987) Biochimica et Biophysica Acta (BBA)-Biomembranes 905(1):162

60. Murtola T, Falck E, Patra M, Karttunen M, Vattulainen I (2004) The Journal of chemical physics 121(18):9156

61. Murtola T, Falck E, Karttunen M, Vattulainen I (2007) The Journal of chemical physics 126(7):02B607

62. Murtola T, Karttunen M, Vattulainen I (2009) The Journal of chemical physics 131(5):08B601

63. Khelashvili G, Pabst G, Harries D (2010) The Journal of Physical Chemistry B 114(22):7524 
64. de Meyer FdrJ-M, Benjamini A, Rodgers JM, Misteli Y, Smit B (2010) The Journal of Physical Chemistry B 114(32):10451

65. Hadley K, McCabe C (2010) Biophysical journal 99(9):2896

66. Hadley K, McCabe C (2012) Soft matter 8(17):4802

67. de Jong DH, Singh G, Bennett WD, Arnarez C, Wassenaar TA, Schäfer LV, Periole X, Tieleman DP, Marrink SJ (2012) Journal of Chemical Theory and Computation 9(1):687

68. Laing C, Baoukina S, Tieleman DP (2009) Physical Chemistry Chemical Physics 11(12):1916

69. Perlmutter JD, Sachs JN (2009) Biochimica et Biophysica Acta (BBA)-Biomembranes 1788(10):2284

70. Khandelia H, Loubet B, Olżyńska A, Jurkiewicz P, Hof M (2014) Soft Matter 10(4):639

71. Duncan SL, Larson RG (2010) Biochimica et Biophysica Acta (BBA)-Biomembranes 1798(9):1632

72. Ogushi F, Ishitsuka R, Kobayashi T, Sugita $Y$ (2012) Chemical Physics Letters 522:96

73. Waheed Q, Tjörnhammar R, Edholm O (2012) Biophysical journal 103(10):2125

74. Zhang Y, Lervik A, Seddon J, Bresme F (2015) Chemistry and physics of lipids 185:88

75. Wang X, Deserno M (2016) The Journal of Physical Chemistry B 120(26):6061

76. Rosetti C, Pastorino C (2012) The Journal of Physical Chemistry B 116(11):3525

77. Davis RS, Sunil Kumar P, Sperotto MM, Laradji M (2013) The Journal of Physical Chemistry B 117(15):4072

78. Chaban VV, Khandelia H (2014) The Journal of Physical Chemistry B 118(38):11145

79. Hakobyan D, Heuer A (2014) PloS one 9(2):e87369

80. Díaz-Tejada C, Ariz-Extreme I, Awasthi N, Hub JS (2015) The journal of physical chemistry letters 6(23):4799

81. Ackerman DG, Feigenson GW (2015) The Journal of Physical Chemistry B 119(11):4240

82. Ferraro M, Masetti M, Recanatini M, Cavalli A, Bottegoni G (2015) RSC Advances 5(47):37102

83. Arnarez C, Marrink S, Periole X (2016) Chemical Science 7(7):4435

84. Daily MD, Olsen BN, Schlesinger PH, Ory DS, Baker NA (2014) Journal of chemical theory and computation 10(5):2137

85. Shen H, Deng M, Wu Z, Zhang J, Zhang Y, Gao C, Cen C (2018) J Chem Theory Comput 14(7):3780

86. Genheden S (2016) Journal of Molecular Graphics and Modelling 63:57

87. Plimpton S (1995) Journal of computational physics 117(1):1

88. Jewett Al, Zhuang Z, Shea J-E (2013) Biophysical Journal 104(2):169a

89. Humphrey W, Dalke A, Schulten K (1996) Journal of molecular graphics 14(1):33

90. Ramırez-Anguita PC, Martinez-Seara H, Giorgino T, Selent J

91. Allen MP, Tildesley DJ (2017) Computer simulation of liquids. Oxford university press,

92. Berendsen HJ, Postma Jv, van Gunsteren WF, DiNola A, Haak J (1984) The Journal of chemical physics 81(8):3684

93. Phillips JC, Braun R, Wang W, Gumbart J, Tajkhorshid E, Villa E, Chipot C, Skeel RD, Kale L, Schulten K (2005) Journal of computational chemistry 26(16):1781

94. Klauda JB, Venable RM, Freites JA, O'Connor JW, Tobias DJ, Mondragon-Ramirez C, Vorobyov I, MacKerell Jr AD, Pastor RW (2010) The journal of physical chemistry B 114(23):7830

95. Lim JB, Rogaski B, Klauda JB (2011) The Journal of Physical Chemistry B 116(1):203

96. Price DJ, Brooks III CL (2004) The Journal of chemical physics 121(20):10096

97. Darden T, York D, Pedersen L (1993) The Journal of chemical physics 98(12):10089

98. Jo S, Lim JB, Klauda JB, Im W (2009) Biophysical journal 97(1):50

99. Miyamoto S, Kollman PA (1992) Journal of computational chemistry 13(8):952

100. Ma Q, Izaguirre JA, Skeel RD (2003) SIAM Journal on Scientific Computing 24(6):1951

101. Grossfield A, Zuckerman DM (2009) Annual reports in computational chemistry 5:23

102. Flyvbjerg H. Error estimates on averages of correlated data. Advances in Computer Simulation: Springer, 1998:88 
103. Darve E, Rodríguez-Gómez D, Pohorille A (2008) The Journal of chemical physics 128(14):144120

104. Hénin J, Chipot C (2004) The Journal of chemical physics 121(7):2904

105. Henin J, Fiorin G, Chipot C, Klein ML (2009) Journal of chemical theory and computation 6(1):35

106. Comer J, Gumbart JC, Hénin J, Lelièvre T, Pohorille A, Chipot C (2014) The Journal of Physical Chemistry B 119(3):1129

107. Taylor J (1997) Introduction to error analysis, the study of uncertainties in physical measurements

108. Falck E, Patra M, Karttunen M, Hyvönen MT, Vattulainen I (2004) Biophysical journal 87(2):1076

109. Edholm O, Nagle JF (2005) Biophysical journal 89(3):1827

110. Voronoï G (1908) Journal für die reine und angewandte Mathematik 134:198

111. Rycroft C (2009)

112. Kessel A, Ben-Tal N, May S (2001) Biophysical Journal 81(2):643

113. Jo S, Rui H, Lim JB, Klauda JB, Im W (2010) The Journal of Physical Chemistry B 114(42):13342

114. Bennett WD, MacCallum JL, Hinner MJ, Marrink SJ, Tieleman DP (2009) Journal of the American Chemical Society 131(35):12714

115. Somerharju PJ, Virtanen JA, Eklund KK, Vainio P, Kinnunen PK (1985) Biochemistry 24(11):2773

116. Tang D, Chong P (1992) Biophysical journal 63(4):903

117. Huang J, Feigenson GW (1999) Biophysical journal 76(4):2142

118. Huang J (2002) Biophysical journal 83(2):1014

119. Liu F, Sugar IP, Chong P (1997) Biophysical journal 72(5):2243

120. Virtanen J, Ruonala M, Vauhkonen M, Somerharju P (1995) Biochemistry 34(36):11568

121. Chong P (1994) Proceedings of the National Academy of Sciences 91(21):10069

122. Zuckermann M, Ipsen J, Mouritsen O (1993) Cholesterol and membrane models CRC Press Inc, Boca Raton, Fla:223

123. Smondyrev AM, Berkowitz ML (1999) Journal of computational chemistry 20(5):531

124. Stockton GW, Smith IC (1976) Chemistry and physics of lipids 17(2-3):251

125. Hung W-C, Lee M-T, Chen F-Y, Huang HW (2007) Biophysical journal 92(11):3960

126. Taylor MG, Smith IC (1980) Biochimica et Biophysica Acta (BBA)-Biomembranes 599(1):140

127. Gershfeld N, Pagano R (1972) The Journal of physical chemistry 76(9):1244

128. Long R, Hruska F, Gesser H, Hsia J, Williams R (1970) Biochemical and biophysical research communications 41(2):321

129. Wydro P, Knapczyk S, Łapczyńska M (2011) Langmuir 27(9):5433

130. Alwarawrah M, Dai J, Huang J (2010) The journal of physical chemistry B 114(22):7516

131. Nagle J (1993) Biophysical journal 64(5):1476

132. Berger O, Edholm O, Jähnig F (1997) Biophysical journal 72(5):2002

133. Dickson CJ, Madej BD, Skjevik ÅA, Betz RM, Teigen K, Gould IR, Walker RC (2014) Journal of chemical theory and computation 10(2):865

134. Zhang Y, Carter JW, Lervik A, Brooks NJ, Seddon JM, Bresme F (2016) Soft matter 12(7):2108

135. Cournia Z, Ullmann GM, Smith JC (2007) The journal of physical chemistry B 111(7):1786

136. Murari R, Murari MP, Baumann WJ (1986) Biochemistry 25(5):1062

137. Karmakar S, Raghunathan V (2005) Physical Review E 71(6):061924

138. Renshaw PF, Janoff AS, Miller KW (1983) Journal of lipid research 24(1):47

139. Vaz W, Almeida PF (1991) Biophysical journal 60(6):1553

140. Falck E, Róg T, Karttunen M, Vattulainen I (2008) Journal of the American Chemical Society 130(1):44

141. Almeida PF, Vaz WL, Thompson T (1992) Biochemistry 31(29):6739

142. Almeida PF, Vaz WL, Thompson T (2005) Biophysical journal 88(6):4434

143. Filippov A, Orädd G, Lindblom G (2003) Biophysical journal 84(5):3079

144. Orädd G, Lindblom G, Westerman PW (2002) Biophysical journal 83(5):2702 
145. Vist MR, Davis JH (1990) Biochemistry 29(2):451

146. Gliss C, Randel O, Casalta H, Sackmann E, Zorn R, Bayerl T (1999) Biophysical journal 77(1):331

147. Lindblom G, Orädd G, Filippov A (2006) Chemistry and physics of lipids 141(1):179

148. Scheidt HA, Huster D, Gawrisch K (2005) Biophysical journal 89(4):2504

149. Lindblom G, Orädd G (2009) Biochimica et Biophysica Acta (BBA)-Biomembranes 1788(1):234

150. Tanaka K, Manning PA, Lau VK, Yu H (1999) Langmuir 15(2):600

151. Filippov A, Orädd G, Lindblom G (2003) Langmuir 19(16):6397

152. Scherfeld D, Kahya N, Schwille P (2003) Biophysical journal 85(6):3758

153. Kahya N, Schwille P (2006) Journal of fluorescence 16(5):671

154. Owicki J, McConnell H (1980) Biophysical journal 30(3):383

155. Essmann U, Berkowitz ML (1999) Biophysical journal 76(4):2081

156. Sackmann E (1995) Handbook of biological physics 1:213

157. Tu K, Klein ML, Tobias DJ (1998) Biophysical journal 75(5):2147

158. Kuo A-L, Wade CG (1979) Biochemistry 18(11):2300

159. Róg T, Pasenkiewicz-Gierula M, Vattulainen I, Karttunen M (2009) Biochimica et Biophysica Acta (BBA)-Biomembranes 1788(1):97

160. Wang Y, Gkeka P, Fuchs JE, Liedl KR, Cournia Z (2016) Biochimica et Biophysica Acta (BBA)Biomembranes 1858(11):2846

161. Jedlovszky P, Mezei M (2003) The Journal of Physical Chemistry B 107(22):5322 